\title{
Shikimate Metabolic Pathway Engineering in Corynebacterium glutamicum
}

\author{
Eunhwi Park ${ }^{1 \dagger}$, Hye-Jin Kim ${ }^{1 \dagger}$, Seung-Yeul Seo ${ }^{2}$, Han-Na Lee ${ }^{2}$, Si-Sun Choi ${ }^{1}$, Sang Joung Lee ${ }^{2}$, and \\ Eung-Soo Kim ${ }^{1 *}$
}

${ }^{1}$ Department of Biological Sciences and Bioengineering, Inha University, Incheon 22212, Republic of Korea ${ }^{2}$ STR Biotech Co., Ltd., Chuncheon 24232, Republic of Korea

\begin{abstract}
Shikimate is a key high-demand metabolite for synthesizing valuable antiviral drugs, such as the anti-influenza drug, oseltamivir (Tamiflu). Microbial-based strategies for shikimate production have been developed to overcome the unstable and expensive supply of shikimate derived from traditional plant extraction processes. In this study, a microbial cell factory using Corynebacterium glutamicum was designed to overproduce shikimate in a fed-batch culture system. First, the shikimate kinase gene (aroK) responsible for converting shikimate to the next step was disrupted to facilitate the accumulation of shikimate. Several genes encoding the shikimate bypass route, such as dehydroshikimate dehydratase (QsuB), pyruvate kinase (Pyk1), and quinate/shikimate dehydrogenase (QsuD), were disrupted sequentially. An artificial operon containing several shikimate pathway genes, including aroE, aroB, aroF, and aroG were overexpressed to maximize the glucose uptake and intermediate flux. The rationally designed shikimate-overproducing C. glutamicum strain grown in an optimized medium produced approximately $37.3 \mathrm{~g} / \mathrm{l}$ of shikimate in 7-L fed-batch fermentation. Overall, rational cell factory design and culture process optimization for the microbial-based production of shikimate will play a key role in complementing traditional plant-derived shikimate production processes.
\end{abstract}

Keywords: Shikimate, metabolic pathway engineering, Corynebacterium, genome editing, fed-batch fermentation

Received: June 4, 2021 Accepted: August 2, 2021

First published online: August 3, 2021

*Corresponding author Phone: 82-32-860-8318 Fax: 82-32-872-4046 E-mail: eungsoo@inha.ac.kr

Supplementary data for this paper are available on-line only at http://jmb.or.kr.

pISSN 1017-7825 elSSN 1738-8872

Copyright(C) 2021 by The Korean Society for Microbiology and Biotechnology

\section{Introduction}

Shikimate is a key metabolic intermediate in the shikimate pathways that are indispensable for maintaining the normal metabolism in plants, animals, and microorganisms. It is also a natural substance with high industrial value as a precursor to oseltamivir, an anti-influenza drug known as Tamiflu $[1,2]$. Shikimate can be used as an intermediate or versatile chiral precursor to synthesize bio-renewable aromatics and stabilize metal nanoparticles. Current methods of shikimate production include extraction from plant star anise (Illicium verum), chemical synthesis, and fermentation of genetically modified microorganisms [1]. Shikimate production by plant-based extraction and chemical synthesis suffers from low yield and high cost, making it difficult to meet the increasing world demand for Tamiflu since the advent of swine and avian influenza [2-4]. Therefore, the fermentation of an engineered microbial strain utilizing renewable resources, such as glucose, would be an alternative sustainable approach [2-7].

Shikimate is typically synthesized by a series of enzyme-led stepwise bioconversions shown in Fig. 1.3-Deoxy$\mathrm{D}$-arabino-heptulosonate-7-phosphate (DAHP) is first produced by the condensation of phosphoenolpyruvate (PEP) and erythrose-4-phosphate (E4P), followed by sequential conversions to 3-dehydroquinate (DHQ), 3dehydroshikimate (DHS), and shikimate. Shikimate is then transformed further to shikimate-3-phosphate by shikimate kinase encoded by aroK. Shikimate-3-phosphate is then transformed into chorismate, which is finally converted to phenylalanine, tyrosine, tryptophan, and other aromatic products. Several metabolic pathwayengineering attempts have been applied to produce useful aromatic compounds and other chemicals derived from the shikimate pathway [8,9]. Examples include salicylic acid (SA) [10], muconic acid [11-14], and 3dehydroshikimate (DHS) [15], which are derived from chorismate and used as precursors in aspirin synthesis.

Corynebacterium glutamicum is a general-regarded-as-safe (GRAS) microorganism that is used industrially for amino acid production. The microorganism has been reported to produce more than two million tones of Lglutamate and one and a half tons of L-lysine annually [16-18]. It has also been used as a host to produce L-arginine [19], L-cysteine [20], anthocyanin [21], hydroxybenzoic acid [22], taurine [23], and 3-hydroxypropionic acid (3-HP) [24], as well as the lignocellulose-based production of fuels and chemicals in biorefineries [25]. Moreover, rationally genome-redesigned C. glutamicum produced approximately $38 \mathrm{~g} / \mathrm{L}$ of muconic acid in 7 -L fed-batch fermentation, suggesting that C. glutamicum could be an ideal bacterial host for an artificial cell factory design [12]. 
Table 1. Strains and plasmids used in this study.

\begin{tabular}{|c|c|c|}
\hline Strain or plasmid & Characteristics & Sources or reference \\
\hline \multicolumn{3}{|c|}{ C. glutamicum ATCC13032 } \\
\hline Inha301 & $\Delta \operatorname{aroK}(\mathrm{NCgl1560)}$ & This study \\
\hline Inha302 & $\Delta a r o K(\mathrm{NCgl1560)} \Delta q s u B(\mathrm{NCg} 10407)$ & This study \\
\hline Inha303 & $\Delta a r o K(\mathrm{NCgl1560}) \Delta q s u B(\mathrm{NCgl0407)} \Delta p y k 1(\mathrm{NCgl} 2008)$ & This study \\
\hline Inha304 & $\Delta a r o K(\mathrm{NCgl1560}) \Delta q s u B(\mathrm{NCgl0407}) \Delta p y k 1(\mathrm{NCgl} 2008) \Delta q s u D(\mathrm{NCgl} 10409)$ & This study \\
\hline Inha305 & Inha304 with pCaroE & This study \\
\hline Inha306 & Inha304 with pEaroE & This study \\
\hline Inha307 & Inha304 with pEC & This study \\
\hline Inha308 & Inha304 with pECB & This study \\
\hline Inha309 & Inha304 with pECBF & This study \\
\hline Inha310 & Inha304 with pECBFG & This study \\
\hline \multicolumn{3}{|l|}{ Plasmid } \\
\hline pK19mobsacB & Vector for the construction of disruption mutants of C. glutamicum & {$[30]$} \\
\hline pSK003 & E. coli-C. glutamicum shuttle vector harboring sod promoter) & This study \\
\hline pCaroE & pSK003 carrying the aroE gene from C. glutamicum ATCC13032 & This study \\
\hline pEaroE & pSK003 carrying the aroE gene from E. coli K-12 & This study \\
\hline $\mathrm{pEC}$ & pEaroE carrying the $q s u C$ gene (NCg10408) from C. glutamicum ATCC13032 & This study \\
\hline $\mathrm{pECB}$ & pEC carrying the aroB gene (NCgl1559) from C. glutamicum ATCC13032 & This study \\
\hline $\mathrm{pECBF}$ & pECB carrying the aroF gene (NCg10950) carrying S188C mutation & This study \\
\hline pECBFG & pECBF carrying the aro $G$ gene from $E$. coli K-12 carrying S180F mutation & This study \\
\hline
\end{tabular}

In the present study, a shikimate high-producing C. glutamicum strain was constructed by shikimate pathway engineering for 7 - $\mathrm{L}$ fed-batch fermentation. These results suggest that the Corynebacterium cell factory design for a shikimate-overproducing strain would be valuable for constructing a microorganism-based high-producing strain for aromatic compounds with industrial value.

\section{Materials and Methods}

\section{Bacterial Strains and Culture Conditions}

Table 1 lists all bacterial strains used in this study. E. coli DH5a was used for genetic manipulation and grown in Luria-Bertani (LB) medium at $37^{\circ} \mathrm{C}$ with the appropriate antibiotics. The C. glutamicum strains were cultivated in a brain heart infusion (BHI) medium and BHIS (BHI medium containing $91 \mathrm{~g} / \mathrm{l}$ sorbitol) at $30^{\circ} \mathrm{C}$. The preparation of shikimate production media and cultivations for C. glutamicum were performed as previously described [12].

\section{Construction of Plasmid and Strains}

Table 1 presents the constructed plasmids, and Table $\mathrm{S} 1$ lists all primer pairs used in this study. For markerless target gene disruption, $\mathrm{pK} 19 \mathrm{mobsacB}$ was used, and the plasmids of $\mathrm{pCaroE}, \mathrm{pEaroE}, \mathrm{pE}, \mathrm{pEC}, \mathrm{pECB}, \mathrm{pECBF}$, and pECBFG were constructed for gene overexpression, which was controlled under the sod promoter. Target gene disruption was verified by colony PCR using each primer set. For overexpression, the aroE genes from C. glutamicum and E. coli were amplified by PCR and inserted into pSK003 using an In-Fusion cloning kit (TaKaRa, Japan), yielding pCaroE and $\mathrm{pEaroE}$, respectively. The $q s u C$, aro $B$ genes were cloned individually into pEaroE to generate $\mathrm{pEC}$ and $\mathrm{pECB}$. A three-way PCR method was used for the site-directed mutagenesis of the aroF gene from C. glutamicum and aroG gene from E. coli, and the $\operatorname{aroF}^{\mathrm{S188C}}$ fragment and EaroG ${ }^{\mathrm{S1} 18 \mathrm{~F}}$ were inserted sequentially into $\mathrm{pECB}$, yielding $\mathrm{pECBF}$ and $\mathrm{pECBFG}$. The constructed plasmids were introduced individually into C. glutamicum strains via electroporation as described [12].

Shikimate and Dehydroshikimate (DHS) Analyses

Cultured broth samples were centrifuged $\left(4^{\circ} \mathrm{C}, 15,000\right.$ RPM for $\left.7 \mathrm{~min}\right)$, and only the supernatant was diluted and purified using a membrane filter (Nylaflo nylon membrane filter) for high-performance liquid chromatography (HPLC). The concentrations of shikimate and DHS were determined by HPLC using an Aminex $\mathrm{HPX}-87 \mathrm{H}$ column (Bio-Rad). The column was heated to $50^{\circ} \mathrm{C}$ to detect shikimate and DHS. The mobile phase was $2.5 \mathrm{mM} \mathrm{H} 2 \mathrm{SO}$, and the flow rate was $0.5 \mathrm{ml} / \mathrm{min}$ for shikimate. Shikimate and DHS were detected at $215 \mathrm{~nm}$ and $236 \mathrm{~nm}$, respectively.

\section{Results and Discussion}

\section{Engineering of the Shikimate Pathway in C. glutamicum ATCC13032}

The shikimate pathway of C. glutamicum was engineered to build a shikimate high-production strain. To construct a Corynebacterium strain that could accumulate shikimate, aroK (NCgl1560) encoding shikimate kinase, which mediates the conversion of shikimate to shikimate-3-phosphate, was first disrupted (named Inha301) and confirmed to produce $0.8 \mathrm{~g} / \mathrm{l}$ of shikimate (Figs. 1 and $2 \mathrm{~A}$ ). $q s u B$ (NCgl0407) was then deleted (named Inha302) to accumulate the key precursor, dehydroshikimate dehydrate (DHS), blocking DHS conversion to protocatechuate (PCA). Approximately, $0.98 \mathrm{~g} / \mathrm{l}$ of DHS was accumulated in the Inha302 strain, 
A

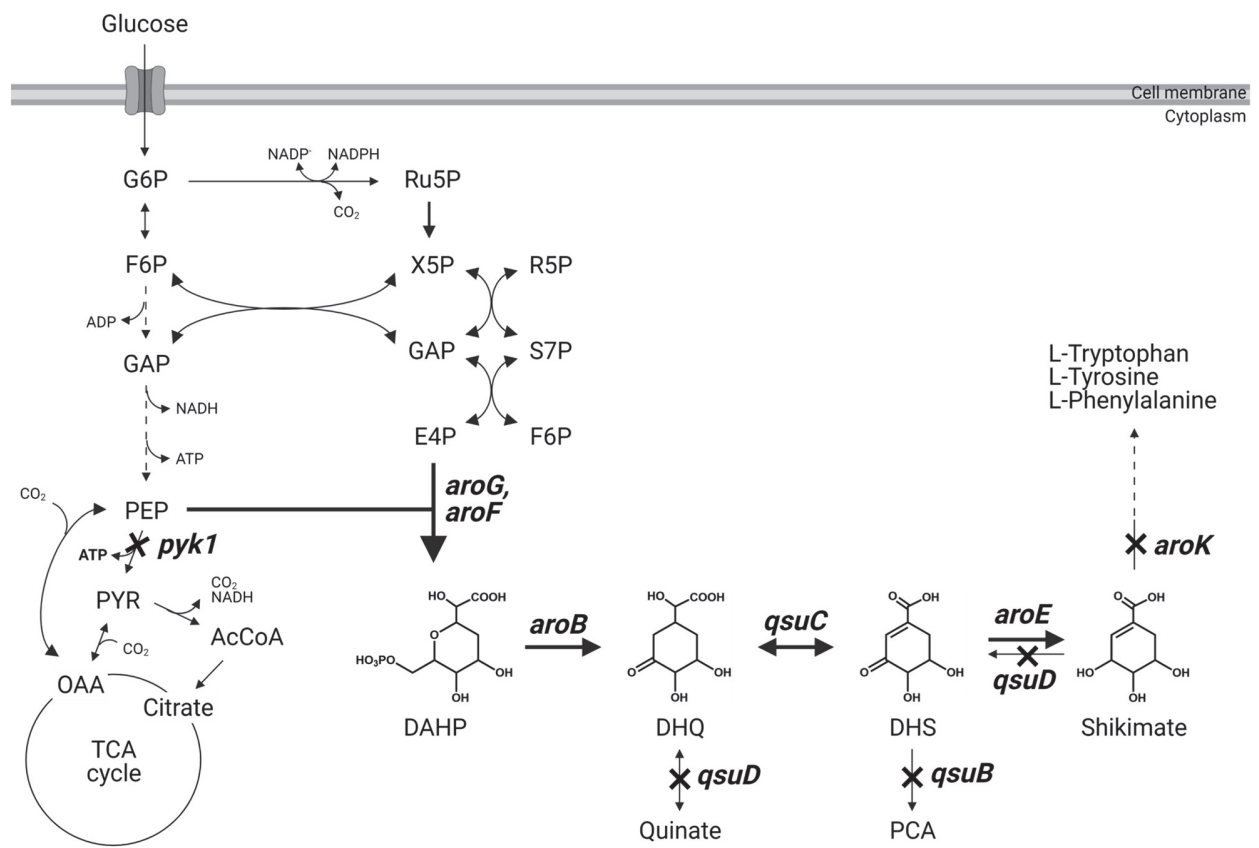

B

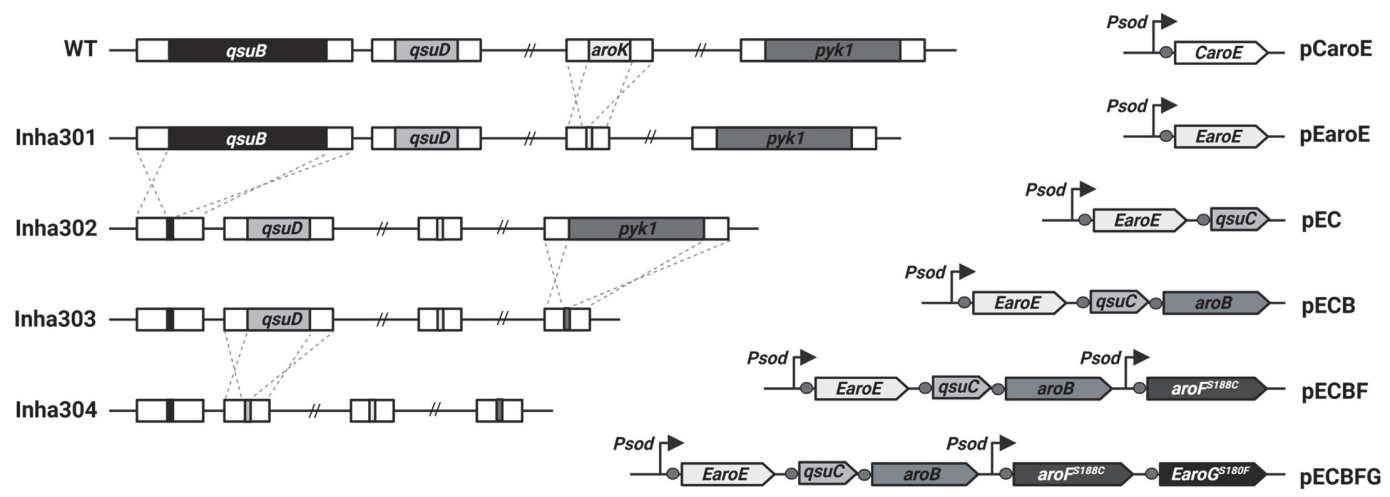

Fig. 1. Pathway engineering strategy for shikimate production in C. glutamicum. (A) Shikimate metabolic pathway in C. glutamicum. The bold arrows and crosses indicate the steps for which corresponding genes were overexpressed and disrupted, individually. The dashed lines represent several catalytic steps. The genes involved in each step are shown in italics. G6P, glucose-6-phosphate; F6P, fructose-6-phosphate; GAP, glyceraldehyde-3-phosphate; PEP, phosphoenolpyruvate; PYR, pyruvate; AcCoA, acetyl-CoA; OAA, oxaloacetate; Ru5P, ribulose-5-phosphate; X5P, xylulose-5-phosphate; R5P, ribose5-phosphate; S7P, sedoheptulose-7-phosphate; E4P, erythrose-4-phosphate; DHAP, 3-deoxy-D-arabinoheptulosanate-7phosphate; DHQ, 3-dehydroquinate; DHS, 3-dehydroshikimate; PCA, protocatechuate. Genes and corresponding enzymes are as follows: pyk1, pyruvate kinase 1; aroF and aroG, DAHP synthase; aroB, 3-dehydroquinate synthase; $q s u C$, dehydroquinate dehydratase; aroE, shikimate dehydrogenase; aroK, shikimate kinase; qsuD, quinate/shikimate dehydrogenase; $q s u B$, dehydroshikimate dehydratase. (B) Gene disruption in C. glutamicum ATCC13032 and plasmid construction. The constructed plasmids were introduced and replicated in Inha304, yielding Inha305, Inha306, Inha307, Inha308, Inha309, and Inha310, respectively. CaroE and EaroE, shikimate dehydrogenase from C. glutamicum ATCC13032 and E. coli K-12, respectively; aroF $F^{S 188 C}$, DAHP synthase carrying S188C mutation; EaroG ${ }^{S 180 F}$, DAHP synthase carrying S180F mutation.

which is 1.97 times higher than $0.5 \mathrm{~g} / \mathrm{l}$ of DHS produced in Inha301 (Fig. 2A). In addition, the pyruvate kinase gene (Pyk1, NCgl2008) was removed (named Inha303) for the build-up of phosphoenolpyruvate (PEP), resulting in $1.33 \mathrm{~g} / \mathrm{l}$ of DHS and $0.95 \mathrm{~g} / \mathrm{l}$ of shikimate (Fig. 2A). Finally, the quinate/shikimate dehydrogenase gene (qsuD) involved in the conversion of 3-dehydroquinate (DHQ) and quinate was deleted (named Inha304) to produce $1.32 \mathrm{~g} / \mathrm{l}$ of shikimate (Fig. 2A). Thus, the sequential elimination of the $\operatorname{aroK}, q s u B$, $p y k 1$, and qusD genes could be inferred by a reasonable pathway engineering method for shikimate high production. 
A
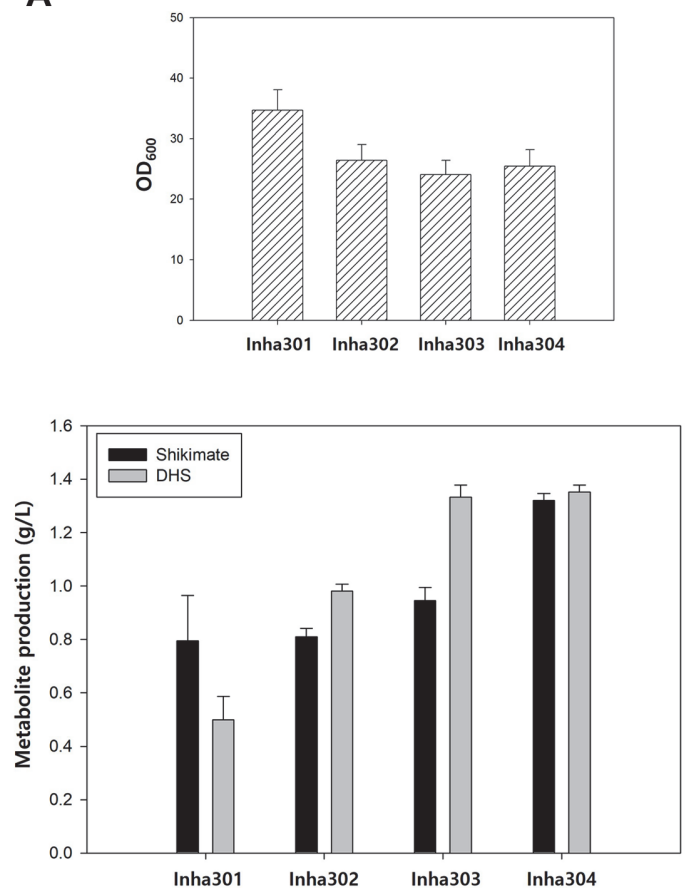

B
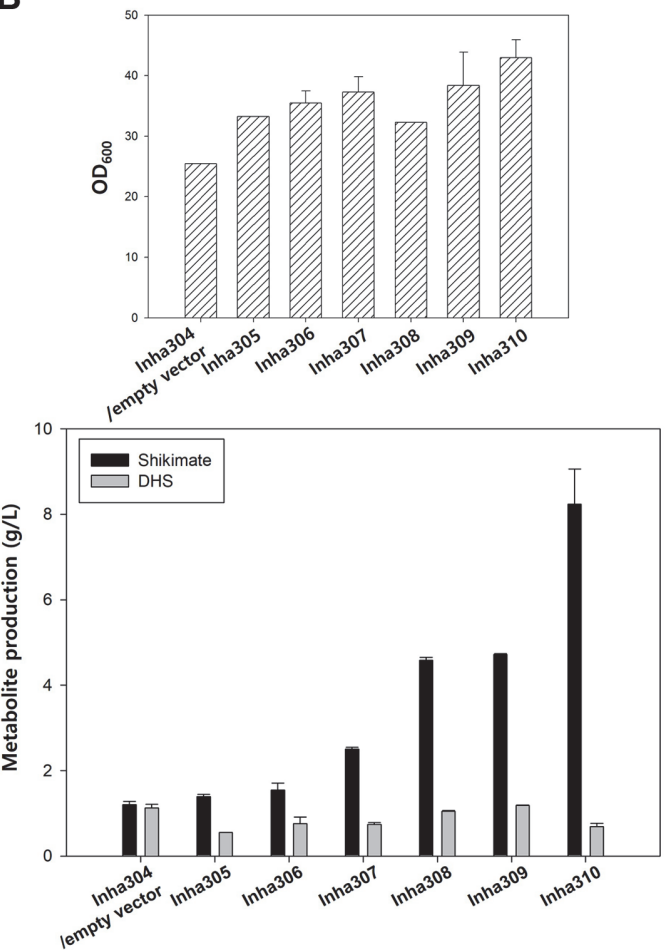

Fig. 2. Metabolite production yield in recombinant C. glutamicum strains. (A) Cell growth (top) and shikimate and dehydroshikimate (DHS) production yield (bottom) in target gene deleted C. glutamicum strains; Inha301, Inha302, Inha303, and Inha304. (B) Cell growth (top) and HPLC analysis of shikimate and dehydroshikimate (DHS) production (bottom) in the Inha304 harboring empty vector, pSK003, and target gene overexpressed, individually; Inha305, Inha306, Inha307, Inha308, Inha309, and Inha310. The values represent the means and standard deviations of duplicate cultivations.

\section{Overexpression of the Shikimate Pathway Genes to Enhance Shikimate Production}

Several key genes involved in shikimate biosynthesis, such as $\operatorname{aroE}, q s u C, \operatorname{aroB}, \operatorname{aroF}$, and aroG, were overexpressed sequentially in the above-constructed four-genes KO stain, Inha304, to maximize the metabolite flux in the shikimate pathway. Five shikimate pathway genes $(a \circ E, q s u C$, aroB, aroF, and aroG) were overexpressed as a single operon under the strong sod promoter, resulting in the simple and rapid construction of a shikimate high-production strain.

Because significant amounts of DHS accumulated in Inha304, as shown in Fig. 2A, the over-expression of AroE, a shikimate dehydrogenase involved in bioconversion from DHS to shikimate, was first attempted in Inha304. For more efficient AroE expression, C. glutamicum-derived aroE (NCg11567) and E. coli-derived aroE were introduced in Inha304 to build Inha305 and Inha306, respectively, and compare the conversion rate to shikimate. As shown in Fig. 2B, $1.55 \mathrm{~g} / \mathrm{l}$ of shikimate was detected in Inha306, which is 1.11 times higher than $1.39 \mathrm{~g} / \mathrm{l}$ of shikimate in Inha305, suggesting that AroE of E. coli might have better enzyme kinetic characteristics. The dehydroquinate dehydrate gene ( $q s u C, \mathrm{NCgl0408)}$ involved in the reaction between 3-dehydroquinate (DHQ) and DHS, and the dehydroquinate synthase gene (aroB, NCgl1559) involved in bioconversion from 3-deoxy-Darabinoheptulosanate to DHQ were sequentially expressed in Inha306, resulting in Inha307 and Inha308, respectively. Inha307 showed a 1.6-fold increase in shikimate production $(2.51 \mathrm{~g} / \mathrm{l})$ compared to Inha306 $(1.55 \mathrm{~g} / \mathrm{l})$, and Inha308 showed a 1.4- and 1.8-fold increase in DHS (1.05 g/l) and shikimate (4.59 g/l) production compared to Inha307, respectively (Fig. 2). Point mutations to the DHAP synthases aroF and aroG, which are involved in the biosynthesis of DHAP from PEP and E4P, have been reported to induce a higher resistance to feedback inhibition $[26,27]$. Hence, the Inha309 strain containing C. glutamicum-derived aroF with a serine to cysteine mutation at the 188 position, and the Inha310 strain containing the E. coli-derived aroG with a serine to phenylalanine mutation at the 180 position, were used. As a result, Inha309 produced $1.19 \mathrm{~g} / \mathrm{l}$ of DHS and $4.72 \mathrm{~g} / \mathrm{l}$ of shikimate, and Inha3 310 exhibited the highest production yields of $0.69 \mathrm{~g} / \mathrm{l}$ of DHS and $8.23 \mathrm{~g} / \mathrm{l}$ of shikimate (Fig. 2B). These results suggest that the pathway engineering strategy described here is a fast and effective approach for shikimate over-production in C. glutamicum.

Fed-Batch Fermentation of Inha310

A $5 \mathrm{~L}$ batch fermentation was performed to calculate the feeding medium flow rate for the fed-batch fermentation of the Inha310 strain. The formula for calculating the feed medium flow rate is as follows. $[F]=$ $\mathrm{Qs}[\mathrm{V}] /[\mathrm{SO}][28]$. Qs, V, and S0 are the initial glucose concentration (g/l)/glucose consumption rate (h), the initial 


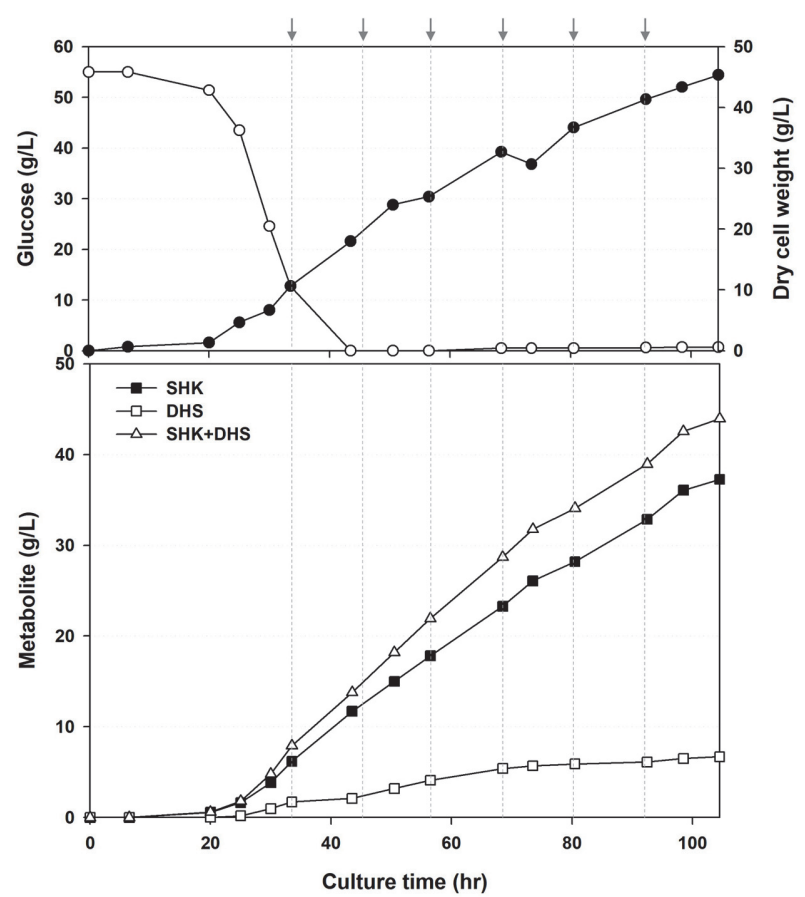

Fig. 3. Time-course profiles of cell growth (Dry cell weight) and glucose concentrations and metabolite production by Inha310 in a 7-L bioreactor. The feeding medium was injected at 33.5, 44.5, 56.5, 68.5, 80.5, and 92.5 h (indicated with gray arrow), at a rate of $0.189,0.246,0.321,0.416,0.548$, and $0.699 \mathrm{ml} / \mathrm{min}$, respectively.

volume of the feeding medium, and glucose concentration of the feeding medium, respectively. Therefore, Inha310 consumed all the glucose in a $2 \mathrm{~L}$ feeding medium containing $55 \mathrm{~g} / \mathrm{l}$ of glucose for $24 \mathrm{~h}$. Hence, the calculated feeding medium flow rate was at least $0.185 \mathrm{ml} / \mathrm{min}$ based on the above formula. After $16-\mathrm{h}$ of $1^{\text {st }}$ seed culture and 6-h of $2^{\text {nd }}$ seed culture, the Inha3 10 culture was inoculated into a $5 \mathrm{~L}$ fermenter. The feeding medium was added at $33.5 \mathrm{~h}$ at a rate of $0.189 \mathrm{ml} / \mathrm{min}$ whenever the glucose concentration decreased below $10 \mathrm{~g} / \mathrm{l}$ during $104.5 \mathrm{~h}$ fermentation. As a result, the Inha3 10 continued to grow during the entire fed-batch fermentation period, and the production of shikimate increased accordingly, finally reaching up to $37.3 \mathrm{~g} / \mathrm{l}$ (Fig. 3).

Similar strategies for shikimate overproduction have been attempted in E. coli and Bacillus subtilis strains, but at a low titer to meet commercial applications [29]. Among the results reported thus far, one of the best titers related to shikimate production is through metabolically engineered C. glutamicum in a growth-arrested cell reaction with $141 \mathrm{~g} / \mathrm{l}$ of shikimate [2]. The shikimate titer should not be compared directly because the fed-batch system described here is quite different from the previously reported cell-arrest culture process. In the future, improvement in the key enzymes through protein engineering, optimization of transcription and translation processes through promoter and ribosomal binding site (RBS) optimization, and stable chromosomal DNA integration will enable the establishment of a more stable shikimate hyper-production strain.

\section{Acknowledgments}

This work was carried out with the support of "Cooperative Research Program for Agriculture Science and Technology Development (Project No. PJ01563901)" Rural Development Administration, Republic of Korea and the National Research Foundation of Korea (NRF), and the Center for Women In Science, Engineering and Technology (WISET-2021-043) Grant funded by the Ministry of Science and ICT(MSIT) under the Program for Returners into R\&D.

\section{Conflict of Interest}

The authors have no financial conflicts of interest to declare.

\section{References}

1. Candeias NR, Assoah B, Simeonov SP. 2018. Production and synthetic modification of shikimic acid. Chem. Rev. 118: 10458-10550.

2. Kogure T, Kubota T, Suda M, Hiraga K, Inui M. 2016. Metabolic engineering of Corynebacterium glutamicum for shikimate overproduction by growth-arrested cell reaction. Metab. Eng. 38: 204-216.

3. Bochkov DV, Sysolyatin SV, Kalashnikov AI, Surmacheva IA. 2012. Shikimic acid: review of its analytical, isolation, and purification techniques from plant and microbial sources. J. Chem. Biol. 5: 5-17.

4. Ghosh S, Chisti Y, Banerjee UC. 2012. Production of shikimic acid. Biotechnol. Adv. 30: 1425-1431.

5. Krämer M, Bongaerts J, Bovenberg R, Kremer S, Müller U, Orf S, et al. 2003. Metabolic engineering for microbial production of shikimic acid. Metab. Eng. 5: 277-283. 
6. Martínez JA, Bolívar F, Escalante A. 2015. Shikimic acid production in Escherichia coli: from classical metabolic engineering strategies to omics applied to improve its production. Front. Bioeng. Biotechnol. 3: 145.

7. Rawat G, Tripathi P, Saxena RK. 2013. Expanding horizons of shikimic acid. Recent progresses in production and its endless frontiers in application and market trends. Appl. Microbiol. Biotechnol. 97: 4277-4287.

8. Li Z, Wang H, Ding D, Liu Y, Fang H, Chang Z, et al. 2020. Metabolic engineering of Escherichia coli for production of chemicals derived from the shikimate pathway. J. Ind. Microbiol. Biotechnol. 47: 525-535.

9. Averesch NJH, Krömer JO. 2018. Metabolic engineering of the shikimate pathway for production of aromatics and derived compounds-present and future strain construction strategies. Front. Bioeng. Biotechnol. 6: 32.

10. Noda S, Shirai T, Oyama S, Kondo A. 2015. Metabolic design of a platform Escherichia coli strain producing various chorismite derivatives. Metab. Eng. 33: 119-129.

11. Lin Y, Sun X, Yuan Q, Yan Y. 2014. Extending shikimate pathway for the production of muconic acid and its precursor salicylic acid in Escherichia coli. Metab. Eng. 23: 62-69.

12. Lee HN, Shin WS, Seo SY, Choi SS, Song JS, Kim JY, et al. 2018 Corynebacterium cell factory design and culture process optimization for muconic acid biosynthesis. Sci. Rep. 8: 18041

13. Choi S, Lee HN, Park E, Lee SJ, Kim ES. 2020. Recent advances in microbial production of cis,cis-muconic acid. Biomolecules 10: 1238 .

14. Fujiwara R, Noda S, Tanaka T, Kondo A. 2020. Metabolic engineering of Escherichia coli for shikimate pathway derivative production from glucose-xylose co-substrate. Nat. Commun. 11:279.

15. Choi SS, Seo SY, Park SO, Lee HN, Song JS, Kim JY, et al. 2019. Cell factory design and culture process optimization for dehydroshikimate biosynthesis in Escherichia coli. Front. Bioeng. Biotechnol. 7: 241.

16. Zahoor A, Lindner SN, Wendisch VF. 2012. Metabolic engineering of Corynebacterium glutamicum aimed at alternative carbon sources and new products. Comput. Struct. Biotechnol. J. 3: e20120004.

17. Hermann T. 2003. Industrial production of amino acids by coryneform bacteria. J. Biotechnol. 104: 155-172.

18. Ikeda M, Takeno S. 2013. Amino acid production by Corynebacterium glutamicum, pp.107-147. In: Yukawa H, Inui M (eds.), Corynebacterium glutamicum. Microbiology Monographs, vol 23. Springer, Berlin, Heidelberg. Germany.

19. Jiang Y, Sheng Q, Wu XY, Ye BC, Zhang B. 2021. L-arginine production in Corynebacterium glutamicum: manipulation and optimization of the metabolic process. Crit. Rev. Biotechnol. 41: 172-185.

20. Kondoh M, Hirasawa T. 2019. L-Cysteine production by metabolically engineered Corynebacterium glutamicum. Appl. Microbiol. Biotechnol. 103: 2609-2619.

21. Zha J, Zang Y, Mattozzi M, Plassmeier J, Gupta M, Wu X, et al. 2018. Metabolic engineering of Corynebacterium glutamicum for anthocyanin production. Microb. Cell Fact. 17: 143

22. Kallscheuer N, Marienhagen J. 2018. Corynebacterium glutamicum as platform for the production of hydroxybenzoic acids. Microb. Cell Fact. 17: 70

23. Joo YC, Ko YJ, You SK, Shin SK, Hyeon JE, Musaad AS, et al. 2018. Creating a new pathway in Corynebacterium glutamicum for the production of taurine as a food additive. J. Agric. Food Chem. 66: 13454-13463.

24. Chang Z, Dai W, Mao Y, Cui Z, Wang Z, Chen T. 2020. Engineering Corynebacterium glutamicum for the efficient production of 3hydroxypropionic acid from a mixture of glucose and acetate via the malonyl-CoA pathway. Catalysts 10: 203

25. Jojima T, Inui M, Yukawa H. 2013. Biorefinery applications of Corynebacterium gluamicum, pp.149-172. In: Yukawa H, Inui M (eds.), Corynebacterium gluamicum. Microbiology Monographs, Vol. 23. Springer, Berlin, Heidelberg. Germany.

26. Liao HF, Lin LL, Chien HR, Hsu WH. 2001. Serine 187 is a crucial residue for allosteric regulation of Corynebacterium glutamicum 3deoxy-Darabino-heptulosonate-7-phosphate synthase. FEMS Microbiol. Lett. 194: 59-64.

27. Ger YM, Chen SL, Chiang HJ, Shiuan D. 1994. A single ser-180 mutation desensitizes feedback inhibition of the phenylalaninesensitive 3-Deoxy-D-Arabino-Heptulosonate 7-Phosphate (DAHP) synthetase in Escherichia coli. J. Biochem. 116: 986-990.

28. Mears L, Stocks SM, Sin G, Gernaey KV. 2017. A review of control strategies for manipulating the feed rate in fed-batch fermentation processes. J. Biotechnol. 245: 34-46.

29. Kogure T, Inui M. 2018. Recent advances in metabolic engineering of Corynebacterium glutamicum for bioproduction of valueadded aromatic chemicals and natural product. Appl. Microbiol. Biotechnol. 102: 8685-8705.

30. Sambrook JF, Maniatis T. 1989. Molecular cloning: A laboratory manual. 2nd Ed. Cold Spring Harbor, New York. 